\title{
On a Trivial Family of Noncommutative Integrable Systems
}

Andrey V. TSIGANOV

St. Petersburg State University, St. Petersburg, Russia

E-mail: andrey.tsiganov@gmail.com

Received October 17, 2012, in final form February 18, 2013; Published online February 22, 2013

http://dx.doi.org/10.3842/SIGMA.2013.015

\begin{abstract}
We discuss trivial deformations of the canonical Poisson brackets associated with the Toda lattices, relativistic Toda lattices, Henon-Heiles, rational Calogero-Moser and Ruijsenaars-Schneider systems and apply one of these deformations to construct a new trivial family of noncommutative integrable systems.
\end{abstract}

Key words: bi-Hamiltonian geometry; noncommutative integrable systems

2010 Mathematics Subject Classification: 37J35; 53D17; 70H06

\section{Introduction}

Let us consider some smooth manifold $M$ with coordinates $x_{1}, \ldots, x_{m}$ and a dynamical system defined by the following equations of motion

$$
\dot{x}_{i}=X_{i}, \quad i=1, \ldots, m \text {. }
$$

We can identify this system of ODE's with the vector field

$$
X=\sum X_{i} \frac{\partial}{\partial x_{i}}
$$

which is a linear operator on a space of the smooth functions on $M$ that encodes the infinitesimal evolution of any quantity

$$
\dot{F}=X(F)=\sum X_{i} \frac{\partial F}{\partial x_{i}}
$$

In Hamiltonian mechanics one of the fundamental axiom is what we can call the energy paradigm that can be stated as follows: "For every mechanical system there is a function defined on its space of states, called mechanical energy or Hamiltonian $H$ of the system, containing all its dynamical information".

According to this paradigm any function $H$ on $M$ generates vector field $X$ describing a dynamical system

$$
X=X_{H}=P d H .
$$

Here $d H$ is a differential of $H$, and $P$ is a bivector on the phase space $M$. By adding some other assumptions we can prove that $P$ is a Poisson bivector. In fact, it is enough to add energy conservation

$$
\dot{H}=X_{H}(H)=(P d H, d H)=0
$$


and compatibility of dynamical evolutions associated with two functions $H_{1,2}$

$$
X_{H_{1}}\left(X_{H_{2}}(F)\right)=X_{H_{2}}\left(X_{H_{1}}(F)\right)+X_{X_{H_{1}}\left(H_{2}\right)}(F),
$$

see $[17,20]$ and references therein.

In bi-Hamiltonian mechanics [25] we are looking for another decomposition of the given vector field $X$,

$$
X=P d H=f_{1} P^{\prime} d H_{1}+\cdots+f_{m} P^{\prime} d H_{m},
$$

by commuting Hamiltonian vector fields

$$
X_{k}=P^{\prime} d H_{k}
$$

generated by integrals of motion $H_{1}, \ldots, H_{m}$ and some Poisson bivector $P^{\prime}$ compatible with $P$. These Poisson bivectors $P^{\prime}$ can be divided into two groups of trivial and nontrivial deformations of canonical Poisson bivector $P$, see [3, 9, 23, 25, 33] and references therein.

Supposing that $P^{\prime}$ is a trivial deformation of canonical Poisson bivector $P$ completely defined by Hamiltonian $H$ we can join the geometry of the phase space $M$ and the energy paradigm. In this case, second Poisson bivector

$$
P^{\prime}=\mathcal{L}_{Y} P
$$

is a Lie derivative of the canonical bivector $P$ along the Liouville vector field

$$
Y=\operatorname{Ad} H,
$$

where $A$ is a 2-tensor field acting on the differential of the Hamiltonian. Remind, that the Lie derivative $P^{\prime}(1.1)$ is a trivial deformation because it is 2-coboundary and simultaneously 2cocycle in the Poisson-Lichnerowicz cohomology defined by canonical Poisson bivector $P[9,23]$.

The main aim of this note is to show 2-tensor fields $A$ associated with some well-known integrable systems and to prove that these tensor fields may be useful to construct new integrable systems. For example, we discuss a new trivial family of integrable noncommutative three dimensional systems, which includes deformations of the rational Calogero-Moser system with three particle interaction.

\section{Integrable systems on cotangent bundles}

Let us consider canonical Poisson bivector on the symplectic manifold $M=T^{*} Q$

$$
P=\sum_{i=1}^{n} \frac{\partial}{\partial q_{i}} \wedge \frac{\partial}{\partial p_{i}}
$$

which is the one mostly used in Hamiltonian mechanics [1, 2]. Here $q_{i}$ are local coordinates describing a point $q$ on a smooth manifold $Q$, and $p_{i}$, the canonical conjugate momenta, are local coordinates describing covectors on such manifold, i.e. points $p$ in the cotangent bundle $T^{*} Q$ of $Q$. The corresponding Poisson bracket looks like

$$
\left\{p_{i}, q_{j}\right\}=\delta_{i j}, \quad\left\{q_{i}, q_{j}\right\}=\left\{p_{i}, p_{j}\right\}=0 .
$$

In local coordinates $x$ on $M$ the Lie derivative of a bivector $P$ along a vector field $Y$ reads as

$$
\left(\mathcal{L}_{Y} P\right)_{i j}=\sum_{k=1}^{\operatorname{dim} M}\left(Y_{k} \frac{\partial P_{i j}}{\partial x_{k}}-P_{k j} \frac{\partial Y_{i}}{\partial x_{k}}-P_{i k} \frac{\partial Y_{j}}{\partial x_{k}}\right)
$$


and the Schouten bracket $[A, B]$ of two bivectors $A$ and $B$ is a trivector with the following entries

$$
[A, B]_{i j k}=-\sum_{m=1}^{\operatorname{dim} M}\left(B_{m k} \frac{\partial A_{i j}}{\partial x_{m}}+A_{m k} \frac{\partial B_{i j}}{\partial x_{m}}+\operatorname{cycle}(i, j, k)\right) .
$$

In our case $M=T^{*} Q, \operatorname{dim} M=2 n$ and $x=(q, p)$.

Let us consider natural Hamilton functions on $M=T^{*} Q$

$$
H=T(q, p)+V(q),
$$

which are the sum of the geodesic Hamiltonian $T$ and potential energy $V(q)$. According to [15, 24, 31, 32], for natural Hamiltonians there is other representation for the vector field $Y(1.2)$

$$
Y=\left(\begin{array}{cc}
\Lambda & 0 \\
0 & \Pi
\end{array}\right)\left(\begin{array}{l}
d q \\
d p
\end{array}\right)
$$

Here we use matrix notation of tensor objects in which, for instance, canonical Poisson bivector $P(2.1)$ looks like

$$
P=\left(\begin{array}{cc}
0 & E \\
-E & 0
\end{array}\right) \text {. }
$$

Here $E$ is a unit matrix.

If $\Pi=0$ and $\Lambda$ is the conformal Killing tensor of gradient type or Yano-Killing tensor on $Q$, one gets second Poisson bivector $P^{\prime}=\mathcal{L}_{Y} P$ associated with Hamilton functions separable in orthogonal coordinate systems on $Q$. In this case eigenvalues of the the Nijenhuis operator

$$
N=P^{\prime} P^{-1},
$$

which is also called the hereditary or recursion operator, are variables of separation. In order to get integrals of motion $H_{k}$ from $N$ we have to extend the initial phase space [18] or to fix separated relations [31].

The new idea is that we can substitute the arbitrary Hamilton function $H(2.4)$ and the 2-tensor field $A$ into the definition (1.1) and try to find the Poisson bivectors $P^{\prime}$ solving the equation

$$
\left[P^{\prime}, P^{\prime}\right]=\left[\mathcal{L}_{\mathrm{Ad} H} P, \mathcal{L}_{\mathrm{Ad} H} P\right]=0,
$$

where $[\cdot, \cdot]$ is the Schouten bracket defined by $(2.3)$. In this case $P^{\prime}$ will be the Poisson bivector compatible with $P$ and we will say that $M=T^{*} Q$ is the bi-Hamiltonian manifold [25]. The next step is a search of integrals of motion for this Hamilton function $H$.

If the recursion operator $N$ at every point has $n$ distinct functionally independent eigenvalues, we can say that $M$ is a regular bi-Hamiltonian manifold. If the recursion operator $N$ does not have this property then we can say that bi-Hamiltonian manifold $M$ is irregular [24, 31]. So, there are three different cases:

1) recursion operator produces the necessary number of integrals of motion;

2) recursion operator generates variables of separation instead of integrals of motion;

3) recursion operator produces only part of the integrals of motion or variables of separation.

In the third case we have to complement the recursion operator with some additional information in order to get integrals of motion. Namely this property allows us to get noncommutative integrable systems, which will be considered in Section 3.

Now let us show a collection of tensor fields $A$ associated with some well-known integrable systems. 


\subsection{Toda lattice}

Let us consider the following tensor field $A$ depending only on $q$ variables

$$
A=\left(\begin{array}{cc}
B+2 D-B^{\top} & 0 \\
0 & B-B^{\top}
\end{array}\right) P
$$

where $B$ is a strictly upper diagonal matrix

$$
B=\left(\begin{array}{ccccc}
0 & 1 & 1 & \cdots & 1 \\
0 & 0 & 1 & \cdots & 1 \\
\vdots & & & \ddots & \vdots \\
& & & 0 & 1 \\
0 & & \cdots & & 0
\end{array}\right)=\sum_{i>j}^{n} e_{i j}
$$

and $D$ is a diagonal matrix

$$
D=\operatorname{diag}\left(q_{1}, q_{2}, \ldots, q_{n}\right)=\sum_{i=1}^{n} q_{i} e_{i i}
$$

Matrices $e_{i j}$ are $n \times n$ with only one non zero $(i j)$ entry, which equals to unit.

Substituting this tensor field $A$ and a natural Hamilton function

$$
H=\sum_{i=1}^{n} p_{i}^{2}+V(q)
$$

into the definition of $P^{\prime}$ one gets a system of equations (2.7) on $V(q)$. One of the partial solutions of this system is the Hamilton function for the open Toda lattice associated with $\mathcal{A}_{n}$ root system

$$
H=\sum_{i=1}^{n} p_{i}^{2}+a \sum_{i=1}^{n-1} e^{q_{i}-q_{i+1}}, \quad a \in \mathbb{R} .
$$

Traces of powers of the corresponding recursion operator $N(2.6)$

$$
H_{k}=\operatorname{tr} N^{k}, \quad k=1, \ldots, n,
$$

are functionally independent constants of motion in bi-involution with respect to both Poisson brackets

$$
\left\{H_{i}, H_{j}\right\}=\left\{H_{i}, H_{j}\right\}^{\prime}=0 .
$$

This Poisson bivector $P^{\prime}$ was found by Das, Okubo and Fernandes $[8,10]$.

In generic case we can use a more complicated tensor field

$$
\widetilde{A}=\left(\begin{array}{cc}
\widetilde{B}+\widetilde{D} & 0 \\
0 & \widetilde{C}
\end{array}\right) P
$$

where entries of $\widetilde{D}$ are linear on $q_{i}$ and $\widetilde{B}$ and $\widetilde{C}$ are numerical matrices. Here $\widetilde{B}$ is an arbitrary matrix, whereas $\widetilde{A}$ and $\widetilde{B}$ satisfy to algebraic equations which may be obtained from (2.7) at $V(q)=0$.

Using this tensor field $\widetilde{A}$ we can get the recursion operators which produce either integrals of motion for the periodic Toda lattice [14] or variables of separation for the Toda lattice [34]. In similar manner we can consider the Toda lattices associated with other classical root systems [31]. 


\subsection{Relativistic Toda lattice}

If we substitute the Hamilton function $H(q, p)$ and the following tensor field $A$

$$
A=\left(\begin{array}{cc}
-B^{\top} & 0 \\
-E & 0
\end{array}\right) P=\left(\begin{array}{cc}
0 & -B^{\top} \\
0 & -E
\end{array}\right)
$$

where $E$ is a unit matrix and $B$ is given by (2.8), into the definition of $P^{\prime}(1.1)$ we will obtain a system of equations on $H$. One of the solutions is the Hamiltonian of the open discrete Toda lattice associated with $\mathcal{A}_{n}$ root system

$$
H=\sum_{i=1}^{n}\left(c_{i}+d_{i}\right)
$$

where $c_{i}$ and $d_{i}$ are the so-called Suris variables

$$
c_{i}=\exp \left(p_{i}-q_{i}+q_{i+1}\right), \quad d_{i}=\exp \left(p_{i}\right), \quad q_{0}=-\infty, \quad q_{n+1}=+\infty .
$$

Traces of powers of the corresponding recursion operator $N(2.9)$ are integrals of motion in bi-involution with respect to both Poisson brackets. Namely this Poisson bivector $P^{\prime}(1.1)$ is discussed in [26, 29].

Remind, that according to [29] there is an equivalence between the relativistic Toda lattice and the discrete time Toda lattice. Namely, substituting

$$
p_{j}=\theta_{j}+\frac{1}{2} \ln \left(\frac{1+\exp \left(q_{j}-q_{j-1}\right)}{1+\exp \left(q_{j+1}-q_{j}\right)}\right)
$$

in (2.10) one gets standard Hamiltonian for the relativistic Toda lattice

$$
H=\sum_{j=1}^{n-1} \exp \left(\theta_{j}\right)\left[\left[1+\exp \left(q_{j}-q_{j-1}\right)\right]\left[1+\exp \left(q_{j+1}-q_{j}\right)\right]\right]^{1 / 2} .
$$

Transformation $\left(\theta_{j} \cdot q_{j}\right) \rightarrow\left(p_{j} \cdot q_{j}\right)$ is a canonical transformation.

As above, two numerical matrices $\widetilde{B}$ and $\widetilde{C}$ in the tensor field

$$
A=\left(\begin{array}{ll}
0 & \widetilde{B} \\
0 & \widetilde{C}
\end{array}\right)
$$

allow us to get recursion operators $N=P^{\prime} P^{-1}$ which generate either integrals of motion for the periodic relativistic Toda lattice or variables of separation [22].

\section{$2.3 \quad H e n o n-H e i l e s$ system}

At $n=2$ we can introduce the following linear in momenta tensor field $A$

$$
A=\left(\begin{array}{cc}
B & 0 \\
0 & C
\end{array}\right) P=\left(\begin{array}{cc}
0 & B \\
-C & 0
\end{array}\right)
$$

where

$$
B=\left(\begin{array}{cc}
2 q_{1} p_{1} & q_{1} p_{2} \\
q_{1} p_{2} & q_{2} p_{2}
\end{array}\right), \quad C=\left(\begin{array}{cc}
f_{1}(q) p_{1}+f_{2}(q) p_{2} & 0 \\
0 & f_{3}(q) p_{1}+f_{4}(q) p_{2}
\end{array}\right) .
$$


Substituting this tensor field $A$ and a natural Hamilton function

$$
H_{1}=p_{1}^{2}+p_{2}^{2}+V(q)
$$

into the definition of $P^{\prime}$ one gets a system of equations (2.7) on $V(q)$ and functions $f_{k}(q)$. The resulting system of PDE's has two partial polynomial solutions

$$
V(q)=c_{1} q_{2}\left(3 q_{1}^{2}+16 q_{2}^{2}\right)+c_{2}\left(2 q_{2}^{2}+\frac{q_{1}^{2}}{8}\right)+c_{3} q_{2}, \quad c_{k} \in \mathbb{R}
$$

and

$$
V(q)=c_{1}\left(q_{1}^{4}+6 q_{1}^{2} q_{2}^{2}+8 q_{2}^{4}\right)+c_{2}\left(q_{1}^{2}+4 q_{2}^{2}\right)+\frac{c_{3}}{q_{2}^{2}} .
$$

Second integrals of motion $\mathrm{H}_{2}=\operatorname{tr} \mathrm{N}^{2}$ are fourth order polynomials in momenta.

So, one gets the Henon-Heiles potential and the fourth order potential [12] as particular polynomial solutions of the equations (2.7) associated with tensor field (2.11).

Using slightly deformed tensor field $A$ we can get the same systems with singular terms [15] and their three-dimensional counterparts [31].

\subsection{Rational Calogero-Moser model}

Following [24] let us consider tensor field $A$, which is proportional to $P$

$$
A=\rho(q, p) P,
$$

where $\rho(q, p)$ is a function on $M$. If

$$
A=\left(p_{1} q_{1}+\cdots+p_{n} q_{n}\right) P, \quad \rho=p_{1} q_{1}+\cdots+p_{n} q_{n},
$$

then equations (2.7) have the following partial solution

$$
H=\frac{1}{2} \sum_{i=1}^{n} p_{i}^{2}+\frac{g^{2}}{2} \sum_{i \neq j}^{n} \frac{1}{\left(q_{i}-q_{j}\right)^{2}},
$$

where $g$ is a coupling constant. It is the Hamilton function of the $n$-particle rational CalogeroMoser model associated with the root system $\mathcal{A}_{n}$.

The corresponding recursion operator $N(2.6)$ generates only a Hamilton function

$$
\operatorname{tr} N^{k}=2 H^{k}, \quad k=1, \ldots, n,
$$

that allows us to identify our phase space $M=\mathbb{R}^{2 n}$ with the irregular bi-Hamiltonian manifold.

In this case $[24,31]$ integrals of motion are polynomial solutions of the equations

$$
P d H=-\frac{1}{k} P^{\prime} d \ln H_{k}, \quad k=1, \ldots, n,
$$

which have two functionally independent solutions for any $k \geq 2$. It is easy to see that the functions

$$
C_{k m}=\frac{H_{m}^{-1 / m}}{H_{k}^{-1 / k}}
$$

are Casimir functions of $P^{\prime}$, i.e. $P^{\prime} d C_{k m}=0$. 
Some solutions of equations (2.14) coincide with the well-known integrals of motion

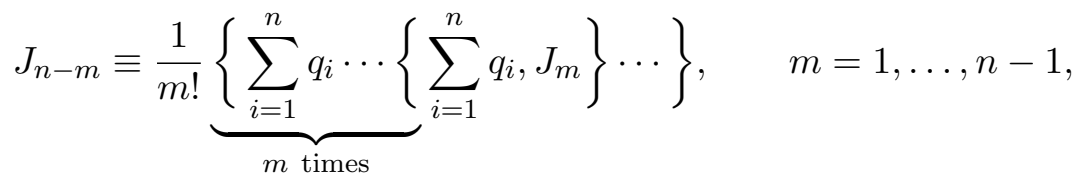

obtained from the conserved quantity

$$
J_{n} \equiv \exp \left(-\frac{g^{2}}{2} \sum_{i \neq j} \frac{1}{\left(q_{i}-q_{j}\right)^{2}} \frac{\partial^{2}}{\partial p_{i} \partial p_{j}}\right) \prod_{k=1}^{n} p_{k}
$$

by taking its successive Poisson brackets with $\sum_{i=1}^{n} q^{i}[11]$. These $n$ solutions, including $J_{2}=H$, are in involution with respect to the Poisson brackets (2.2).

Other $n-1$ functionally independent solutions of (2.14),

$$
K_{m}=m g_{1} J_{m}-g_{m} J_{1}, \quad g_{m}=\frac{1}{2}\left\{\sum_{i=1}^{n} q_{j}^{2}, J_{m}\right\}, \quad m=2, \ldots, n,
$$

are not in involution with respect to the canonical Poisson bracket defined by (2.1) [11].

\subsection{Rational Ruijsenaars-Schneider model}

Let us consider tensor field $A$, which is proportional to canonical bivector $P$

$$
A=\left(q_{1}+\cdots+q_{n}\right) P, \quad \rho=q_{1}+\cdots+q_{n} .
$$

In this case equations (2.7) have the following partial solutions

$$
J_{k}=\frac{1}{k !} \operatorname{tr} L^{k}, \quad k= \pm 1, \pm 2, \ldots, \pm n,
$$

where $L$ is the Lax matrix of the Ruijsenaars-Schneider model

$$
L=\sum_{i, j=1}^{n} \frac{\gamma}{q_{i}-q_{j}+\gamma} b_{j} e_{i j}, \quad b_{k}=e^{p_{k}} \prod_{j \neq k}\left(1-\frac{\gamma^{2}}{\left(q_{k}-q_{j}\right)^{2}}\right)^{1 / 2} .
$$

As above recursion operator produces only the Hamilton function. It is easy to prove that traces of powers of the Lax matrix $L$ (2.16) satisfy to the following relations

$$
P d J_{ \pm 2}=-\frac{1}{k} P^{\prime} d \ln J_{k}, \quad k= \pm 1, \ldots, \pm n,
$$

instead of the standard Lenard-Magri relations [25, 30]. Moreover, similar to the CalogeroMoser system, there are other solutions $K_{m}$ of these equations (2.17), which are described in [4].

Remind, that the so-called principal Ruijsenaars-Schneider Hamiltonian has the form

$$
H_{R S}=\frac{1}{2}\left(J_{1}+J_{-1}\right)=\sum_{k=1}^{n}\left(\cosh 2 p_{k}\right) \prod_{j \neq k}\left(1-\frac{\gamma^{2}}{\left(q_{k}-q_{j}\right)^{2}}\right)^{1 / 2}
$$

and that the rational Ruijsenaars-Schneider system is in duality with the corresponding variant of the trigonometric Sutherland system, see [4] and references therein.

We want to highlight that for all integrable systems listed in $[24,30,31,32]$ the second Poisson bivector $P^{\prime}(1.1)$ is a Lie derivative of the canonical Poisson bivector $P$ along the vector field $Y=\operatorname{Ad} H(1.2)$, where tensor field $A$ usually has a very simple form.

In the next section we show that such simple tensor fields $A$ may be useful to search for new integrable systems. 


\section{Noncommutative integrable systems}

The extreme rarity of integrable dynamical systems makes the quest for them all the more exciting. We want to apply tensor fields $A$ to partial solution of this problem. Below we present a method to construct a new family of three dimensional noncommutative integrable systems.

Let us consider natural Hamilton function on $M=\mathbb{R}^{2 n}$

$$
H=\sum_{i=1}^{n} p_{i}^{2}+V\left(q_{1}, \ldots, q_{n}\right)
$$

and bivector $A$ associated with the rational Calogero-Moser system (2.12)

$$
A=\left(p_{1} q_{1}+\cdots+p_{n} q_{n}\right) P
$$

where $P$ is canonical Poisson bivector (2.1), (2.5).

In previous section we have discussed partial solutions of the equations $(2.7)$, here we want to discuss their complete solution.

Proposition 1. The Lie derivative of $P(2.1)$ along the vector field $Y$

$$
P^{\prime}=\mathcal{L}_{Y} P, \quad Y=\left(p_{1} q_{1}+\cdots+p_{n} q_{n}\right) P d H
$$

is a Poisson bivector compatible with $P$ if and only if

$$
H=\sum_{i=1}^{n} p_{i}^{2}+\frac{1}{q_{1}^{2}} F\left(\frac{q_{2}}{q_{1}}, \frac{q_{3}}{q_{1}}, \ldots, \frac{q_{n}}{q_{1}}\right) .
$$

Here $F$ is an arbitrary homogeneous function of zero degree function depending on the homogeneous coordinates

$$
x_{1}=\frac{q_{2}}{q_{1}}, \quad x_{2}=\frac{q_{3}}{q_{1}}, \ldots, \quad \ldots x_{n-1}=\frac{q_{n}}{q_{1}} .
$$

The definition of the homogeneous coordinates may be found in [13]. Proof is a straightforward calculation of the Schouten bracket (2.7).

It is easy to see that some Hamilton functions separable in spherical coordinates and Hamilton functions for the rational Calogero-Moser systems associated with the $A_{n}, B_{n}, C_{n}$ and $D_{n}$ root systems have the form (3.2).

We got accustomed to believing that the notion of two compatible Poisson structures $P$ and $P^{\prime}$ allows us to get the appropriate integrable systems [15, 25, 30, 31, 32]. In our case recursion operator $N=P^{\prime} P^{-1}$ reproduces only the Hamilton function

$$
\operatorname{tr} N^{k}=2(2 H)^{k} \text {. }
$$

It allows us to identify our phase space $M=\mathbb{R}^{2 n}$ with the irregular bi-Hamiltonian manifold $[25,31]$, but simultaneously it makes the use of standard constructions of the integrals of motion impossible.

We do not claim that all the Hamilton functions (3.2) are integrable because we do not have an explicit construction of the necessary number of integrals of motion. Nevertheless, even in generic case there is one additional integral of motion.

Proposition 2. The following second order polynomial in momenta

$$
C=\left(p_{1} q_{1}+\cdots+p_{n} q_{n}\right)^{2}-\left(q_{1}^{2}+\cdots+q_{n}^{2}\right) H
$$

is a Casimir function of $P^{\prime}$, i.e. $P^{\prime} d C=0$. 
Consequently we have

$$
\{H, C\}=0 .
$$

It is enough for integrability at $n=2$ when we get Hamilton functions

$$
H=p_{1}^{2}+p_{2}^{2}+\frac{1}{q_{1}^{2}} F\left(\frac{q_{2}}{q_{1}}\right)
$$

separable in polar coordinates on the plane.

At $n>3$ we can make some assumptions on the form of the additional integrals of motion. For instance, let us postulate that our dynamical system is invariant with respect to translations, i.e. that there is a linear in momenta integral of motion

$$
H_{\text {post }}=p_{1}+\cdots+p_{n}, \quad\left\{H, H_{\text {post }}\right\}=0 .
$$

It leads to the additional restriction on the form of the proper Hamilton functions (3.2)

$$
H=\sum_{i=1}^{n} p_{i}^{2}+\frac{1}{\left(q_{2}-q_{1}\right)^{2}} G\left(\frac{q_{3}-q_{2}}{q_{2}-q_{1}}, \frac{q_{4}-q_{3}}{q_{2}-q_{1}}, \ldots, \frac{q_{n}-q_{n-1}}{q_{1}-q_{2}}\right)
$$

which generate bi-Hamiltonian vector fields

$$
X=P d H=P^{\prime} d \ln H_{\text {post }}^{-1}
$$

equipped with the four integrals of motion

$$
H_{1}=H_{\text {post }}, \quad H_{2}=H, \quad H_{3}=C, \quad H_{4}=\left\{H_{1}, C\right\}
$$

with the linearly independent differentials $d H_{i}$. According to the Euler-Jacobi theorem [19] it is enough for integrability by quadratures at $n=3$.

Remind that the Euler-Jacobi theorem [19] states that a system of $N$ differential equations

$$
\dot{x}_{i}=X_{i}\left(x_{1}, \ldots, x_{N}\right), \quad i=1, \ldots, N,
$$

possessing the last Jacobi multiplier $\mu$ (invariant measure) and $N-2$ independent first integrals is integrable by quadratures. In our case $N=6$, we have four independent integrals of motion (3.4) and $\mu=1$.

So, at $n=3$ the following Hamilton functions

$$
H_{2}=p_{1}^{2}+p_{2}^{2}+p_{3}^{3}+\frac{1}{\left(q_{2}-q_{1}\right)^{2}} G\left(\frac{q_{3}-q_{2}}{q_{2}-q_{1}}\right)
$$

labelled by functions $G$ generate integrable by quadratures Hamiltonian equations of motion (3.3)-(3.5). Because

$$
\begin{array}{lll}
\left\{H_{1}, H_{2}\right\}=0, & \left\{H_{1}, H_{3}\right\}=H_{4}, \quad & \left\{H_{1}, H_{4}\right\}=2 H_{1}^{2}-6 H_{2}, \\
\left\{H_{2}, H_{3}\right\}=0, & \left\{H_{2}, H_{4}\right\}=0, & \left\{H_{4}, H_{3}\right\}=4 H_{1} H_{3}
\end{array}
$$

we have noncommutative integrable systems with respect to the canonical Poisson bracket, see, for instance, [21] and references therein.

Of course, in the center of momentum frame, the total linear momentum of the system is zero $H_{1}=0$ and we have three integrals of motion $H_{2}, H_{3}$ and $H_{4}$ in the involution that is enough for integrability at $n=3$ and $n=4$. 
On the other hand, Hamilton functions (3.6) define superintegrable systems in the Liouville sense

$$
\left\{H_{i}, H_{j}\right\}^{\prime}=0, \quad i, j=1, \ldots, 4
$$

with respect to the second Poisson bracket $\{\cdot, \cdot\}^{\prime}$ associated with the Poisson tensor $P^{\prime}(3.1)$. If we put

$$
G(x)=g^{2}\left(1+\frac{1}{x^{2}}+\frac{1}{(1+x)^{2}}\right),
$$

we can obtain a well-known Hamiltonian for the rational Calogero-Moser system (2.13)

$$
H_{\mathrm{CM}}=\sum_{i=1}^{3} p_{i}^{2}+\frac{g^{2}}{\left(q_{2}-q_{1}\right)^{2}}+\frac{g^{2}}{\left(q_{3}-q_{2}\right)^{2}}+\frac{g^{2}}{\left(q_{3}-q_{1}\right)^{2}} .
$$

In this case there are other polynomial integrals of motion (2.14) and other Casimir functions of $P^{\prime}$ (2.15). This system was separated by Calogero [7] in cylindrical coordinates in $\mathbb{R}^{3}$. Being a superintegrable system it is actually separable in four other types of coordinate systems [5]. These variables of separation may be easily found using either the generalised Bertrand-Darboux theorem [35] or methods of the bi-Hamiltonian geometry [16]. Remind, that variables of separation are eigenvalues of the Killing tensor $K$ satisfying equation

$$
K d V=0,
$$

where $V$ is potential part of the Hamiltonian $H$.

Any additive deformation of this function $G(x)$ leads to the integrable additive deformation of the rational Calogero-Moser system, for instance, if

$$
\widetilde{G}(x)=G(x)+\frac{a}{x},
$$

then one gets an integrable system with the three-particle interaction

$$
\widetilde{H}_{\mathrm{CM}}=H_{\mathrm{CM}}+\frac{a}{\left(q_{1}-q_{2}\right)\left(q_{2}-q_{3}\right)} .
$$

For this Hamilton function we couldn't find any polynomial in momenta integrals of motion except $H_{1}, H_{3}$ and $H_{4}(3.4)$ ). Moreover, we couldn't get variables of separation using the standard (regular) methods such as generalised Bertrand-Darboux theorem [35] and bi-Hamiltonian algorithm discussed in [16]. Namely, in contrast with the case $a=0$ at $a \neq 0$ the Killing tensor $K$ satisfying (3.8) has only functionally dependent eigenvalues.

At $n=3$ in order to get rational Calogero-Moser systems associated with other classical root systems and their deformations we can postulate an existence of the fourth order integral of motion

$$
H_{\text {post }}=\sum_{i \neq j}^{n} p_{i}^{2} p_{j}^{2}+\sum_{k} f_{k}(q) p_{k}^{2}+g(q),
$$

with some unknown functions $f_{k}(q)$ and $g(q)$. However we do not have an exhaustive classification as of yet.

In generic case at $n \geq 3$ we can use other hypotheses about additional integrals of motion commuting with $H(3.2)$. 
Of course, construction of such integrable systems is trivial and closely related with construction of the group invariant solutions of partial differential equations through imposing side conditions $[27,28]$. Remind, we can look for solution $W(q)$ of the Hamilton-Jacobi equation

$$
H(p, q)=\sum_{i=1}^{n}\left(\frac{\partial W}{\partial q_{i}}\right)^{2}+W\left(q_{1}, \ldots, q_{n}\right)=\mathcal{E}, \quad p_{j}=\frac{\partial W}{\partial q_{j}},
$$

up to the side condition

$$
\mathcal{S}(q, p)=0 .
$$

If $\{H, \mathcal{S}\}=f(q, p) \mathcal{S}$ then this side condition is consistent with $H$ and the corresponding integrals of motion are defined modulo $\mathcal{S}=0$, i.e.

$$
\left\{H, H_{k}\right\}=g_{k}(q, p) \mathcal{S} .
$$

Here $f$ and $g_{k}$ are some functions on phase space and $W(q)$ is the so-called characteristic Hamilton function.

In our case the side condition is related with the transition to the center of momentum frame

$$
\mathcal{S}=H_{\text {post }} \equiv p_{1}+p_{2}+p_{3}=0,
$$

which is always consistent with the Hamilton function (3.6) and we have three integrals of motion $H_{2}, H_{3}$ and $H_{4}(3.4)$ in involution by modulo $\mathcal{S}=0$ (3.7). Construction of the variables of separation for the Hamilton-Jacobi equation with a side conditions is discussed in [6].

In quantum case we can consider the Schrödinger equation

$$
H \Psi=\mathcal{E} \Psi, \quad H=\Delta+V\left(q_{1}, \ldots, q_{n}\right),
$$

where $\Delta$ is the Laplace-Beltrami operator on $M=\mathbb{R}^{2 n}$, and study solution of this equation that also satisfies a side condition

$$
\mathcal{S} \Psi=0 .
$$

The consistency condition for the existence of nontrivial solutions $\Psi$ is a standard

$$
[H, \mathcal{S}]=f \mathcal{S} .
$$

In this case linear differential operator $K$ will be a symmetry operator for $H$ modulo $\mathcal{S} \Psi=0$ if

$$
[H, K]=g \mathcal{S} .
$$

Here $f$ and $g$ are some linear partial differential operators, see examples and discussion in [6].

We assume that the quantum counterpart of $H$ (3.6) could be embedded in this generic scheme.

\section{Conclusion}

We have demonstrated that the trivial deformations of the canonical Poisson bracket associated with the well-known integrable systems have a very simple form defined by some 2-tensor field $A$ acting on the differential of the Hamilton function. We have shown a collection of examples and also proven that such tensor fields may be useful for searching new integrable by quadratures dynamical systems. For example, we have proven the noncommutative integrability of a new generalisation of the rational Calogero-Moser system with three particle interaction.

In fact, we propose a new form for the old content and believe that this unification is a next step in creating the invariant and rigorous geometric theory of integrable systems on regular and irregular bi-Hamiltonian manifolds. 


\section{Acknowledgements}

We would like to thank E.G. Kalnins, W. Miller, Jr. and G. Rastelli for useful discussion on noncommutative integrable systems. The study was supported by the ministry of education and science of Russian Federation, project 07.09.2012 no. 8501, grant no. 2012-1.5-12-000-1003016.

\section{References}

[1] Abraham R., Marsden J.E., Foundations of mechanics, 2nd ed., Benjamin/Cummings Publishing Co. Inc., Reading, Mass., 1978.

[2] Arnol'd V.I., Mathematical methods of classical mechanics, Graduate Texts in Mathematics, Vol. 60, 2nd ed., Springer-Verlag, New York, 1989.

[3] Arsie A., Lorenzoni P., On bi-Hamiltonian deformations of exact pencils of hydrodynamic type, J. Phys. A: Math. Theor. 44 (2011), 225205, 31 pages, arXiv:1101.0167.

[4] Ayadi V., Fehér L., Görbe T.F., Superintegrability of rational Ruijsenaars-Schneider systems and their action-angle duals, J. Geom. Symmetry Phys. 27 (2012), 27-44, arXiv:1209.1314.

[5] Benenti S., Chanu C., Rastelli G., The super-separability of the three-body inverse-square Calogero system, J. Math. Phys. 41 (2000), 4654-4678.

[6] Broadbridge P., Chanu C.M., Miller Jr. W., Solutions of Helmholtz and Schrödinger equations with side condition and nonregular separation of variables, SIGMA 8 (2012), 089, 31 pages, arXiv:1209.2019.

[7] Calogero F., Solution of a three-body problem in one dimension, J. Math. Phys. 10 (1969), 2191-2196.

[8] Das A., Okubo S., A systematic study of the Toda lattice, Ann. Physics 190 (1989), 215-232.

[9] Degiovanni L., Magri F., Sciacca V., On deformation of Poisson manifolds of hydrodynamic type, Comm. Math. Phys. 253 (2005), 1-24, nlin.SI/0103052.

[10] Fernandes R.L., On the master symmetries and bi-Hamiltonian structure of the Toda lattice, J. Phys. A: Math. Gen. 26 (1993), 3797-3803.

[11] Gonera C., Nutku Y., Super-integrable Calogero-type systems admit maximal number of Poisson structures, Phys. Lett. A 285 (2001), 301-306, nlin.SI/0105056.

[12] Grammaticos B., Dorizzi B., Ramani A., Hamiltonians with high-order integrals and the "weak-Painlevé" concept, J. Math. Phys. 25 (1984), 3470-3473.

[13] Griffiths P., Harris J., Principles of algebraic geometry, Wiley Classics Library, John Wiley \& Sons Inc., New York, 1994.

[14] Grigoryev Yu.A., Tsiganov A.V., On the Darboux-Nijenhuis variables for the open Toda lattice, SIGMA 2 (2006), 097, 15 pages, nlin.SI/0701004.

[15] Grigoryev Yu.A., Tsiganov A.V., Separation of variables for the generalized Henon-Heiles system and system with quartic potential, J. Phys. A: Math. Theor. 44 (2011), 255202, 9 pages, arXiv:1012.0468.

[16] Grigoryev Yu.A., Tsiganov A.V., Symbolic software for separation of variables in the Hamilton-Jacobi equation for the $L$-systems, Regul. Chaotic Dyn. 10 (2005), 413-422, nlin.SI/0505047.

[17] Ibort A., The geometry of dynamics, Extracta Math. 11 (1996), 80-105.

[18] Ibort A., Magri F., Marmo G., Bihamiltonian structures and Stäckel separability, J. Geom. Phys. 33 (2000), $210-228$.

[19] Jacobi C.G.J., Vorlesungen über dynamik, G. Reimer, Berlin, 1884.

[20] Jost R., Poisson brackets (an unpedagogical lecture), Rev. Modern Phys. 36 (1964), 572-579.

[21] Khesin B., Tabachnikov S., Contact complete integrability, Regul. Chaotic Dyn. 15 (2010), 504-520, arXiv:0910.0375.

[22] Kuznetsov V.B., Tsiganov A.V., Separation of variables for the quantum relativistic Toda lattices, J. Math. Sci. 80 (1994), 1802-1810, hep-th/9402111.

[23] Lichnerowicz A., Les variétés de Poisson et leurs algèbres de Lie associées, J. Differential Geometry 12 (1977), 253-300. 
[24] Maciejewski A.J., Przybylska M., Tsiganov A.V., On algebraic construction of certain integrable and superintegrable systems, Phys. D 240 (2011), 1426-1448, arXiv:1011.3249.

[25] Magri F., Casati P., Falqui G., Pedroni M., Eight lectures on integrable systems, in Integrability of Nonlinear Systems (Pondicherry, 1996), Lecture Notes in Phys., Vol. 495, Springer, Berlin, 1997, 256-296.

[26] Oevel W., Fuchssteiner B., Zhang H., Ragnisco O., Mastersymmetries, angle variables, and recursion operator of the relativistic Toda lattice, J. Math. Phys. 30 (1989), 2664-2670.

[27] Olver P.J., Rosenau P., Group-invariant solutions of differential equations, SIAM J. Appl. Math. 47 (1987), 263-278.

[28] Ovsiannikov L.V., Group analysis of differential equations, Academic Press Inc., New York, 1982.

[29] Suris Y.B., On the bi-Hamiltonian structure of Toda and relativistic Toda lattices, Phys. Lett. A 180 (1993), 419-429.

[30] Tempesta P., Tondo G., Generalized Lenard chains, separation of variables, and superintegrability, Phys. Rev. E 85 (2012), 046602, 11 pages, arXiv:1205.6937.

[31] Tsiganov A.V., On bi-integrable natural Hamiltonian systems on Riemannian manifolds, J. Nonlinear Math. Phys. 18 (2011), 245-268, arXiv:1006.3914.

[32] Tsiganov A.V., On natural Poisson bivectors on the sphere, J. Phys. A: Math. Theor. 44 (2011), 105203, 21 pages, arXiv:1010.3492.

[33] Tsiganov A.V., On the Poisson structures for the nonholonomic Chaplygin and Veselova problems, Regul. Chaotic Dyn. 17 (2012), 439-450.

[34] Tsiganov A.V., On two different bi-Hamiltonian structures for the Toda lattice, J. Phys. A: Math. Theor. 40 (2007), 6395-6406, nlin.SI/0701062.

[35] Waksjö C., Rauch-Wojciechowski S., How to find separation coordinates for the Hamilton-Jacobi equation: a criterion of separability for natural Hamiltonian systems, Math. Phys. Anal. Geom. 6 (2003), 301-348. 